\title{
BIO_09 - Evaluation of liposomal formulation carrying interference RNA for the treatment of
} breast tumors

Ana Beatriz Teixeira Frederico ${ }^{1 *}$; Danielle Regina de Almeida de Brito e Cunha ${ }^{1}$; Juliana Gil Melgaço ${ }^{1}$; Luciana Neves Tubarão; Beatriz Ferreira de Carvalho Patricio ${ }^{2}$; Helvécio Vinicius Antunes Rocha ${ }^{2}$; Tatiana Martins Tilli³; Ana Paula Dinis Ano Bom ${ }^{1}$.

${ }^{1}$ Fiocruz/Bio-Manguinhos;

${ }^{2}$ Fiocruz/Farmanguinhos;

${ }^{3}$ Fiocruz/CDTS.

Introduction: Cancer is the second leading cause of death from diseases in the world. Only in 2018, this disease was responsible for about 9.6 million deaths, where breast cancer is the tumor type with the highest incidence and mortality in the female population worldwide. Taking into account the adverse effects of current treatments, in addition to low efficiency and specificity, it is evident the need to develop new therapies aimed at greater specificity and less side effects.

Objective: This work aims to develop cationic liposomes that carry interference RNA (siRNA) molecules for silencing target genes in breast tumors.

Methodology: The selection of targets was previously outlined by our research group based on data from the interactome and transcriptome of seven tumor cell lines and one non-tumor cell line. The development of the liposome was carried out by forming a lipid film of phospholipid and cholesterol followed by hydration of the it and subsequent extrusion. The particle physicochemical characterization was evaluated by dynamic light scattering (DLS) and zeta potential; and their biological effects were assessed. First, we evaluated the effect of delivery formulations (empty cationic liposome, in the presence or absence of Polyethylene glycol - PEG) on cell viability in MCF-7 tumor cell line (human breast adenocarcinoma, Luminal A), MDA-MB-231 (human breast adenocarcinomas, triple negative) and HEP-G2 (human hepatocarcinoma). Flow cytometry methodology was used to evaluate gene silencing, where cells were treated with siRNA-carrying liposomes in different concentrations for 48 hours.

Results: We performed bioassays at different times and concentrations, that shows greater cell viability in the presence of liposomes with PEG when compared in the absence of it, however, both demonstrated viability greater than $50 \%$. Then, we proceed with the evaluation of protein silencing in flow cytometry after treatment with siRNA carrier liposomes in the presence or absence of PEG, in different concentrations for 48 hours. The silencing evaluation showed a decrease in the expression of two target proteins in cells treated with liposomes containing RNAi for these two targets.

Conclusion: The liposomes were successfully obtained. The evaluation of the silencing of two target proteins revealed a decrease in their expression in relation to the control, indicating the efficiency of siRNA delivery through liposome system. Thus, our results indicate a promising profile of the target genes under study for the development of an innovative therapy in the treatment of breast cancer.

Keywords: liposome; RNAi; breast cancer 